\title{
Inefficiency and classical communication bounds for conversion between partially entangled pure bipartite states
}

\author{
Ben Fortescue and Hoi-Kwong Lo \\ Center for Quantum Information and Quantum Control, Department of Electrical \& Computer Engineering and Department of Physics, \\ University of Toronto, Toronto, Ontario, Canada M5S $3 G 4$ \\ (Received 15 February 2005; published 28 September 2005)
}

\begin{abstract}
We derive lower limits on the inefficiency and classical communication costs of dilution between two-term bipartite pure states that are partially entangled. We first calculate explicit relations between the allowable error and classical communication costs of entanglement dilution using a previously described protocol, then consider a two-stage dilution from singlets with this protocol followed by some unknown protocol for conversion between partially entangled states. Applying overall lower bounds on classical communication and inefficiency to this two-stage protocol, we derive bounds for the unknown protocol. In addition we derive analogous (but looser) bounds for general pure states.
\end{abstract}

DOI: 10.1103/PhysRevA.72.032336

PACS number(s): 03.67.Mn

\section{INTRODUCTION}

In the resource model of quantum-information processing, bipartite pure-state entanglement is regarded as a resource which is fungible (i.e., it can be efficiently converted between different forms) in the asymptotic limit. One can concentrate entanglement (convert from many states of low entanglement to fewer states of higher entanglement) or dilute entanglement (the reverse process). The standard unit of entanglement is the ebit, the entanglement of an EinstenRosen-Podolsky (EPR) pair, singlet, or maximally entangled state of the form

$$
\frac{1}{\sqrt{2}}(|00\rangle+|11\rangle)_{A B}
$$

shared between two parties Alice and Bob.

Asymptotically reversible protocols for concentration and dilution to and from singlets were given by Bennett, Bernstein, Popescu, and Schumacher (BBPS) [1]. These are reversible in that the entanglement loss due to the process is only a negligible fraction of the original amount of entanglement-using them one can convert $M$ copies of an EPR state $\Phi$ to $N$ copies of a pure state $\psi$ such that $M / N$ $\rightarrow S(\psi)$ as $N \rightarrow \infty$, where $S(\psi)$ is the entanglement of $\psi$, defined as $-\operatorname{Tr} \rho \log _{2} \rho$ where $\rho=|\psi\rangle\langle\psi|$. One can likewise perform the reverse process with asymptotically negligible loss of entanglement.

However, these protocols may require a nontrivial amount of classical communication. While the BBPS protocol for concentration requires no classical communication, that for dilution requires 2 bits of classical communication per ebit diluted. If all protocols for dilution required 2 classical bits of communication per ebit, then entanglement could not truly be regarded as a fungible resource. For instance, starting with partially entangled states, superdense coding could not provide any saving in classical communication cost. Fortunately, Lo and Popescu [2] showed that dilution of roughly $N S(\psi)$ singlets to $N$ copies of a state $\psi$ could be done with only $O(\sqrt{N})$ bits of classical communication in the large $N$ limit, compared to $2 \mathrm{~N}$ bits via the BBPS protocol. It was later shown by Harrow and Lo [3] and independently by Hayden and Winter [4] that there is a lower bound on the classical communication cost of this dilution of $O(\sqrt{N})$ bits and a lower bound on the inefficiency of this dilution (i.e., the number of ebits lost in the dilution) of $O(\sqrt{N})$ bits also (so the process can still be asymptotically efficient with inefficiency per ebit $\rightarrow 0$ ). Specifically they showed that, for dilution from $p$ singlets to $N$ copies of a state $\psi$ with probability of success $2^{-s}$ using $c$ bits of classical communication,

$$
\begin{gathered}
p \geqslant N S(\psi)+\alpha_{\psi} \sqrt{N}, \\
c+s \geqslant \alpha_{\psi} \sqrt{N},
\end{gathered}
$$

where for $|\psi\rangle\langle\psi|$ having eigenvalues $p_{i}$,

$$
\alpha_{\psi}^{2} \equiv \sum_{i} p_{i}\left[\log _{2} p_{i}+S(\psi)\right]^{2} \quad\left(\alpha_{\psi}>0\right)
$$

These results all apply solely to dilution from singlets to partially entangled states and do not address the situation of conversion between multiple copies of two different partially entangled states, e.g., from roughly $N S\left(\psi_{2}\right) / S\left(\psi_{1}\right)$ copies of an entangled state $\psi_{1}$ to $N$ copies of an entangled state $\psi_{2}$. Some results regarding dilutions of this kind follow immediately from the above results-it is clear from [1] that one can perform such a dilution asymptotically efficiently, by efficient concentration from the initial state to singlets followed by efficient dilution from singlets to the final state. Likewise there is an upper limit of $O(\sqrt{N})$ bits of classical communication required for such a dilution, since one can always concentrate to singlets (at zero classical communication cost) and then dilute via the Lo-Popescu protocol at a cost of $O(\sqrt{N})$ bits.

However, it is not immediately clear what, if any, lower bound applies to the inefficiency and classical communication cost of converting between partially entangled statesthe above results do not forbid there being a cost of, e.g., 


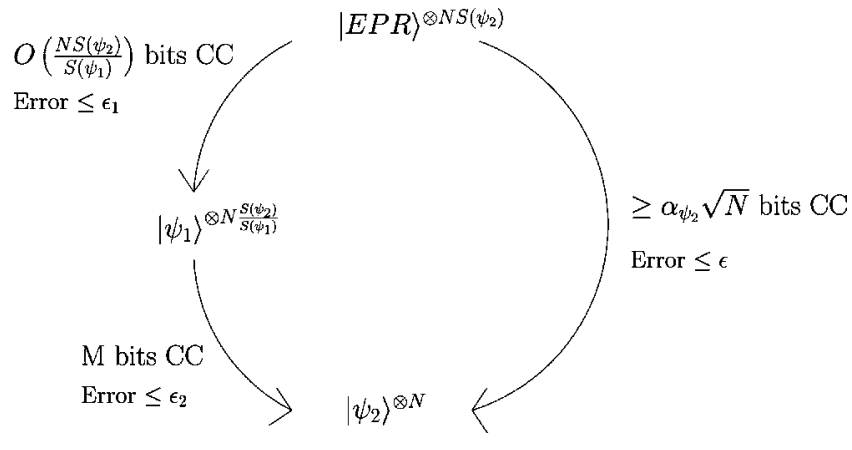

FIG. 1. Classical communication (CC) costs of entanglement dilution via a two-stage protocol, the Lo-Popescu protocol followed by some unknown protocol for dilution between partially entangled states. The right-hand side gives the Harrow-Lo bound on classical communication for the whole process.

zero or $O(\log N)$, though intuitively one would expect there to be a nonzero cost in, for example, diluting from a state very close to an EPR state.

In this paper, we do the following.

(1) We construct explicit bounds on the classical communication cost and inefficiency for conversion between partially entangled states.

(2) We give explicit examples of partially entangled states that require $O(\sqrt{N})$ bits of classical communications between their interconversion and likewise for inefficiency.

(3) To do so, we have also worked out the dependence of the coefficient of the $O(\sqrt{N})$ term in the classical communication cost on the error (as measured in trace distance) in the Lo-Popescu protocol for entanglement dilution.

\section{OUR METHOD}

In this paper, we study the entanglement manipulation process from roughly $N S\left(\psi_{2}\right) / S\left(\psi_{1}\right)$ copies of a partially entangled state $\psi_{1}$ to $N$ copies of another partially entangled state $\psi_{2}$. We are interested in its classical communication cost and also its inefficiency (i.e., loss in entanglement). Initially we will focus on the case where $\psi_{1}$ has a Schmidt number of only 2 . In other words, $\psi_{1}=a_{1}|00\rangle+b_{1}|11\rangle$.

The method we use was proposed in [3] and its idea is to consider a two-stage dilution. First, roughly $N S\left(\psi_{2}\right)$ singlets are diluted to roughly $N S\left(\psi_{2}\right) / S\left(\psi_{1}\right)$ copies of a state $\psi_{1}$ via the Lo-Popescu protocol, using $\beta \sqrt{N}$ bits of classical communication for some $\beta$. These are then converted to roughly $N$ copies of a state $\psi_{2}$ via some unknown protocol, using $m$ bits of classical communication. This two-stage process is one way of implementing the dilution from singlets to $\psi_{2}$ and must therefore obey the lower bound of Harrow and Lo [3] and Hayden and Winter [4]. The method is illustrated graphically in Fig. 1. Thus we have that

$$
\beta \sqrt{N}+m \geqslant \alpha_{\psi_{2}} \sqrt{N}
$$

therefore

$$
m \geqslant\left(\alpha_{\psi_{2}}-\beta\right) \sqrt{N}
$$

Hence we can derive a lower limit on the classical communication cost of the conversion from $\psi_{1}$ to $\psi_{2}$ if we can calculate the coefficient $\beta$. This is not derived in [2] and we do it here.

\section{CLASSICAL COMMUNICATION COST IN THE LO- POPESCU PROTOCOL}

A priori, the parameter $\beta$ may depend on the target state in question, the allowable error, and/or the success probability of the Lo-Popescu protocol. However the explicit dependence of $\beta$ on these parameters is not derived in [2]. In this section we work out this dependence in detail.

The Lo-Popescu protocol works briefly as follows. $N$ copies of a desired partially entangled state $\psi$ may be expressed as

$$
\psi^{N}=\Phi^{d} \otimes \Delta+\epsilon_{L P}
$$

where those terms making up $u_{1} \equiv \Phi^{d} \otimes \Delta$ have high degeneracy $2^{d}$ where $d=N S(\psi)-O(\sqrt{N})$ in their Schmidt coefficients and those in the error term $\epsilon_{L P}$ have low degeneracy. In diluting from singlets $\Phi$, all that needs to be teleported is one-half of the residual state $\Delta$ with dimension $2^{O(\sqrt{N})}$ and thus only $O(\sqrt{N})$ bits are required compared to the $2 N$ bits that would be needed to teleport the entire state $\psi^{N}$ to perform the dilution naively.

\section{A. Error size}

The size of the coefficient on $\sqrt{N}$ in the classical communication cost depends on the allowable error $\epsilon_{L P}$. We derive this dependency for the general two-term case $|\psi\rangle=a|00\rangle$ $+b|11\rangle$, for which we have that the degeneracy of coefficients in $\left|\psi^{\otimes N}\right\rangle$ containing $k$ a's is $\left(\begin{array}{c}N \\ k\end{array}\right)$. We define $p=|a|^{2}, q$ $=|b|^{2}=1-p$, and, as in [2], we define the typical set as those coefficients for which

$$
2^{N S(\psi)-\gamma \sqrt{N}} \leqslant\left(\begin{array}{l}
N \\
k
\end{array}\right) \leqslant 2^{N S(\psi)+\gamma \sqrt{N}}
$$

for some coefficient $\gamma$. We wish to find what range of $k$ this corresponds to.

Using Stirling's approximation for $N$ !, we have that

$$
\lim _{N \rightarrow \infty}\left(\begin{array}{l}
N \\
k
\end{array}\right)=2^{N S_{b}(k / N)}
$$

where $S_{b}$ is the binary entropy function

$$
\begin{gathered}
S_{b}(x)=-x \log _{2} x-(1-x) \log _{2}(1-x), \\
\frac{d S_{b}}{d x}=-\log _{2} x+\log _{2}(1-x), \\
\frac{d x}{d S_{b}}=\frac{1}{-\log _{2} x+\log _{2}(1-x)} .
\end{gathered}
$$

Hence the typical set corresponds to a range 


$$
S(\psi)-\gamma / \sqrt{N} \leqslant S_{b}(k / N) \leqslant S(\psi)+\gamma / \sqrt{N} .
$$

We consequently calculate $d(k / N) / d S_{b}=(1 / N) d k / d S_{b}$ and can thus write $k\left(S_{b}\right)$ as a Taylor expansion to first order in $S_{b}$ (higher orders are negligible as $N \rightarrow \infty$ ) about the point $S_{b}(k / N)=S(\psi), k=N p$. We find that for the typical set (assuming henceforth that $p<q$; this is without loss of generality except in the case $p=q=0.5$ for which the above procedure is not valid but which physically represents having singlets as the target state, in which case no dilution is required)

$$
N p-\frac{\gamma \sqrt{N}}{\log _{2}(q / p)} \leqslant k \leqslant N p+\frac{\gamma \sqrt{N}}{\log _{2}(q / p)} .
$$

Thus the typical set is defined by these values of $k$ with the remaining terms forming the atypical set. The contribution $\epsilon_{L P_{1}}$ of the atypical set to the total weight of the state $\left\langle\psi^{\otimes N} \mid \psi^{\otimes N}\right\rangle$ is

$$
\sum_{k_{\text {atypical }}}\left(\begin{array}{c}
N \\
k
\end{array}\right) p^{k} q^{N-k}
$$

a binomial distribution for which in the large $N$ limit we can use the Gaussian approximation as given in, e.g., [5]:

$$
\left(\begin{array}{l}
N \\
k
\end{array}\right) p^{k} q^{N-k} \sim \frac{e^{-(k-N p)^{2} / 2 N p q}}{\sqrt{2 \pi N p q}}
$$

giving

$$
\begin{aligned}
\epsilon_{L P_{1}} & =2 \times \frac{1}{\sqrt{2 \pi N p q}} \int_{-\infty}^{N p-\gamma \sqrt{N} / \log _{2}(q / p)} e^{-(k-N p)^{2} / 2 N p q} d k \\
& =2 \times\left(1-\frac{1}{\sqrt{2 \pi}} \int_{-\infty}^{\gamma / \alpha} e^{-x^{2} / 2} d x\right)
\end{aligned}
$$

where $\alpha=\sqrt{p q} \log _{2}(q / p)=\sqrt{\sum_{i} p_{i}\left[\log _{2} p_{i}+S(\psi)\right]^{2}}$, the same $\alpha(\psi)$ defined in [3].

We are not aware of any analytical expression for Eq. (13), but it may be bounded [5] as

$$
\begin{aligned}
& \sqrt{\frac{2}{\pi}}\left(\frac{1}{(\gamma / \alpha)}-\frac{1}{(\gamma / \alpha)^{3}}\right) e^{-(1 / 2)(\gamma / \alpha)^{2}} \\
& \quad \leqslant \epsilon_{L P_{1}} \leqslant \sqrt{\frac{2}{\pi}}\left(\frac{1}{(\gamma / \alpha)}\right) e^{-(1 / 2)(\gamma / \alpha)^{2}} .
\end{aligned}
$$

However, the error term $\epsilon_{L P}$ in Eq. (6) does not consist only of atypical terms - as described in [2], in order to ensure a large common degeneracy for the terms in $u_{1}$ of $2^{N S(\psi)-\omega \sqrt{N}}$ for some chosen $\omega>\gamma$, certain terms from the typical set need to be assigned to the error term.

Claim 1. The contribution $\epsilon_{L P_{2}}$ to $\left\langle\epsilon_{L P} \mid \epsilon_{L P}\right\rangle$, due to those typical terms which are grouped in the error term in Lo and Popescu's protocol, goes to zero in the limit that the number of copies $N$ goes to infinity. Hence in this limit $\left\langle\epsilon_{L P} \mid \epsilon_{L P}\right\rangle$ $=\epsilon_{L P_{1}}$

Proof. As shown in [2], these typical terms have a degen- eracy $\leqslant 2^{N S(\psi)-\omega \sqrt{N}}$, and thus their contribution $\epsilon_{L P_{2}}$ to $\left\langle\epsilon_{L P} \mid \epsilon_{L P}\right\rangle$ satisfies

$$
\epsilon_{L P_{2}} \leqslant 2^{N S(\psi)-\omega \sqrt{N}} \sum_{k_{\text {typical }}} p^{k} q^{N-k} .
$$

Approximating the sum to an integral in the limit of large $N$,

$$
\begin{aligned}
\epsilon_{L P_{2}} & \leqslant 2^{N S(\psi)-\omega \sqrt{N}} q^{N} \int_{N p-\gamma \sqrt{N} / \log _{2}(q / p)}^{N p+\gamma \sqrt{N} / \log _{2}(q / p)}(p / q)^{k} d k \\
& =\frac{2^{-\omega \sqrt{N}}}{\ln (q / p)}\left[2^{\gamma \sqrt{N}}-2^{-\gamma \sqrt{N}}\right] \\
& \rightarrow \frac{2^{-(\omega-\gamma) \sqrt{N}}}{\ln (q / p)} \operatorname{as} N \rightarrow \infty .
\end{aligned}
$$

Thus in the asymptotic limit we can take $\left\langle\epsilon_{L P} \mid \epsilon_{L P}\right\rangle=\epsilon_{L P_{1}}$ and choose $\omega=\gamma+\delta$ for positive $\delta \rightarrow 0$, giving a common degeneracy of $2^{N S(\psi)-\gamma \sqrt{N}}$ to the terms in $u_{1}$; thus $u_{1}$ can be expressed as $\Phi^{d} \otimes \Delta$ where $d=N S(\psi)-\gamma \sqrt{N}$.

Claim 2. The error term $\epsilon_{L P_{1}}$ in Lo and Popescu's protocol is bounded by Eq. (14) whose upper bound may be inverted to give

$$
\frac{\gamma}{\alpha} \leqslant \sqrt{W\left(\frac{2}{\pi \epsilon_{L P_{1}}^{2}}\right)}
$$

where $W()$ is the Lambert $W$ function [6].

Proof. It is clear from Eq. (14) that for a given $\epsilon_{L P_{1}}$, the largest value of $\gamma / \alpha$ corresponds to the inverse of the upper bound. The form of the inverse follows from the definition of $W(x)$ that $W(x) e^{W(x)}=x$.

\section{B. Classical communication cost}

$\operatorname{Sch}\left(u_{1}\right)$, defined as the Schmidt number of $u_{1}$, is simply $\Sigma_{k_{\text {typical }}}\left(\begin{array}{l}N \\ k\end{array}\right)$. We are not aware of any analytical expression for this sum, but it may be bounded [7] as

$$
A_{0} 2^{N S_{b}(1 / g)} \leqslant \operatorname{Sch}\left(u_{1}\right) \leqslant A_{1} 2^{N S_{b}(1 / g)}
$$

where $1 / g=\left[p+\gamma / \sqrt{N} \log _{2}(q / p)\right]$ and $A_{0}$ and $A_{1}$ scale at most polynomially with $N$.

Thus asymptotically we find

$$
\operatorname{Sch}\left(u_{1}\right)=A 2^{N S(\psi)+\gamma \sqrt{N}}
$$

for some $A$ scaling at most polynomially with $N$. Since $u_{1}$ $=\Phi^{d} \otimes \Delta$ we have that

$$
\operatorname{Sch}(\Delta)=A 2^{N S(\psi)+\gamma \sqrt{N}-d}=A 2^{2 \gamma \sqrt{N}} .
$$

It was shown in [8] that production of an entangled state via quantum teleportation can be performed with half the classical communication cost of naive teleportation of the state $\Delta$. Hence the asymptotic classical communication cost of diluting from singlets to $N$ copies of a state $\psi$ via the Lo-Popescu protocol is $\frac{1}{2} \times 2 \times 2 \gamma \sqrt{N}=2 \gamma \sqrt{N}$ bits. 


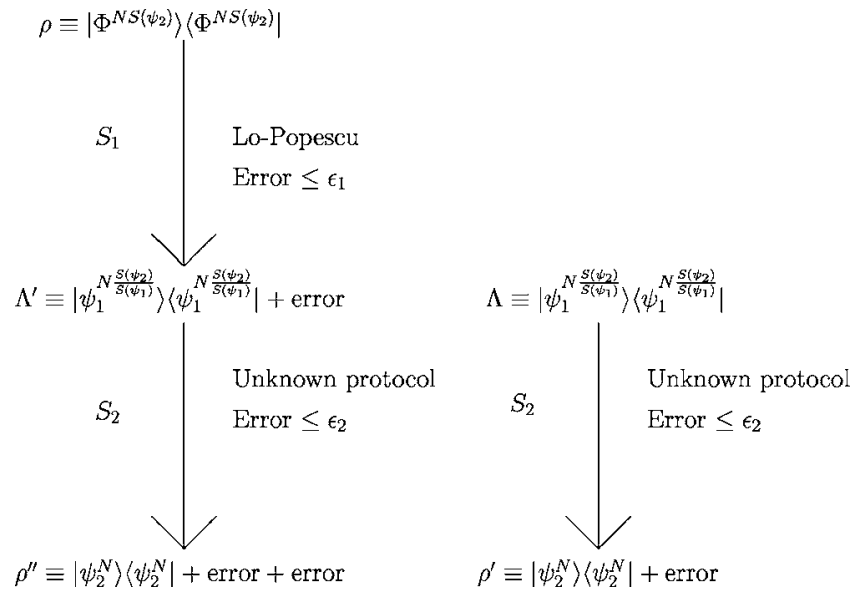

FIG. 2. Errors for different stages of the two-stage dilution.

\section{RELATION BETWEEN PROTOCOL ERRORS}

We first define the trace distance between states with density matrices $\sigma$ and $\rho, D(\sigma, \rho) \equiv \operatorname{Tr}|\sigma-\rho|$.

In our two-stage protocol, we start with roughly $N S\left(\psi_{2}\right)$ singlets with an overall density matrix $\sigma$. We then dilute using the Lo-Popescu protocol to a state $\Lambda^{\prime}$ which is close to the desired state of $N S\left(\psi_{2}\right) / S\left(\psi_{1}\right)$ copies of $\psi_{1}$, with density matrix $\Lambda$. Finally we dilute $\Lambda^{\prime}$ via some unknown protocol to $\rho^{\prime \prime}$, close to the desired state $\rho$ of $N$ copies of a state $\psi_{2} . \rho^{\prime \prime}$ differs from $\rho$ due to the errors introduced at both dilution stages-we in addition define $\rho^{\prime}$ as the state that one would acquire from the unknown protocol if $\Lambda$ was used as the input state.

We define the upper bounds of the errors in the protocols as follows:

$$
\begin{gathered}
D\left(\Lambda, \Lambda^{\prime}\right) \leqslant \epsilon_{1} \quad \text { (error in the Lo-Popescu protocol) } \\
D\left(\rho^{\prime}, \rho\right) \leqslant \epsilon_{2} \quad \text { (error in unknown protocol) } \\
D\left(\rho^{\prime \prime}, \rho\right) \leqslant \epsilon \quad \text { (overall error in two-stage protocol) }
\end{gathered}
$$

The above is illustrated in Figure 2.

Since our final bounds will depend only on $\epsilon_{2}$ we are free to choose our allowable error $\epsilon_{1}$ in the Lo-Popescu dilution as long as it is consistent with the requirement of the Harrow-Lo bound (under "Definition of error" in Theorem 1 of [3]) that

$$
D\left(\rho, \rho^{\prime \prime}\right) \leqslant 0.01 \text {. }
$$

We can describe the Lo-Popescu dilution as a tracepreserving operation $S_{1}$ and likewise the unknown protocol as a trace-preserving operation $S_{2}$, so that

$$
\Lambda^{\prime}=S_{1} \sigma, \quad \rho^{\prime \prime}=S_{2} \Lambda^{\prime}=S_{2} S_{1} \sigma, \quad \rho^{\prime}=S_{2} \Lambda .
$$

From the triangle inequality (see, for example, [9]) we have that, since trace-preserving quantum operations are contractive (applying $S_{2}$ cannot increase the trace distance) with just a "hence" before Eq. (24)

$$
\begin{aligned}
\epsilon_{2} & =D\left(\rho^{\prime \prime}, \rho\right)=D\left(S_{2} S_{1} \sigma, \rho\right) \leqslant D\left(S_{2} S_{1} \sigma, S_{2} \Lambda\right)+D\left(S_{2} \Lambda, \rho\right) \\
& \leqslant D\left(S_{1} \sigma, \Lambda\right)+D(\Lambda, \rho)=D\left(S_{1} \sigma, \Lambda\right)+\epsilon_{2},
\end{aligned}
$$

$$
D\left(S_{1} \sigma, \Lambda\right)+\epsilon_{2}=D\left(\Lambda, \Lambda^{\prime}\right)+\epsilon_{2}=\epsilon_{1}+\epsilon_{2} .
$$

Hence

$$
D\left(\rho, \rho^{\prime \prime}\right) \leqslant \epsilon_{1}+\epsilon_{2} .
$$

In performing a two-stage dilution, we are free to choose the $\epsilon_{1}$ for our Lo-Popescu protocol. However, in order to derive inefficiency and classical communication bounds for the second stage we must be able to apply the Harrow-Lo bound and hence must satisfy Eq. (23). But given this restriction, we would like $\epsilon_{1}$ to be as large as possible so that $\gamma / \alpha_{\psi_{1}}$ is small and hence the inefficiency and classical communication bounds are restrictive; $\epsilon_{1} \rightarrow 0$ would give $\gamma / \alpha \rightarrow \infty$ and hence no meaningful bound (since the Lo-Popescu classical communication cost and inefficiency would then be very large for almost all $\psi_{1}$ and hence satisfy the Harrow-Lo bound without the need for any classical communication or inefficiency from the second stage of the dilution). Thus for given $\epsilon_{2}<0.01$, from Eq. (24) we satisfy Eq. (23) by setting

$$
\epsilon_{1}=0.01-\epsilon_{2} \text {. }
$$

In the Lo-Popescu protocol the (normalized) state obtained is $\left|\Phi^{d} \otimes \Delta+\epsilon_{L P}\right\rangle\left\langle\Phi^{d} \otimes \Delta+\epsilon_{L P}\right|$ and the (normalized) desired state is $\left|\Phi^{d} \otimes \Delta\right\rangle\left\langle\Phi^{d} \otimes \Delta\right| /\left(1-\epsilon_{L P_{1}}\right)$. Thus we find, from claim 1 ,

$$
\begin{aligned}
& \operatorname{Tr}\left|\Lambda^{\prime}-\Lambda\right|=\operatorname{Tr} \mid \frac{\left|\Phi^{d} \otimes \Delta\right\rangle\left\langle\Phi^{d} \otimes \Delta\right|}{1-\epsilon_{L P_{1}}} \\
& -\left|\Phi^{d} \otimes \Delta+\epsilon_{L P}\right\rangle\left\langle\Phi^{d} \otimes \Delta+\epsilon_{L P}||\right. \\
& \left.=\operatorname{Tr}\left|\frac{\epsilon_{L P_{1}}\left|\Phi^{d} \otimes \Delta\right\rangle\left\langle\Phi^{d} \otimes \Delta\right|}{1-\epsilon_{L P_{1}}}-\right| \Phi^{d} \otimes \Delta\right\rangle\left\langle\epsilon_{L P}\right| \\
& -\left|\epsilon_{L P_{1}}\right\rangle\left\langle\Phi^{d} \otimes \Delta|-| \epsilon_{L P}\right\rangle\left\langle\epsilon_{L P}||\right. \\
& \left.=\operatorname{Tr}\left|\frac{\epsilon_{L P_{1}}\left|\Phi^{d} \otimes \Delta\right\rangle\left\langle\Phi^{d} \otimes \Delta\right|}{1-\epsilon_{L P_{1}}}-\right| \epsilon_{L P}\right\rangle\left\langle\epsilon_{L P}||\right. \\
& =\left|\epsilon_{L P_{1}}\right|+\left|-\epsilon_{L P_{1}}\right|=2 \epsilon_{L P_{1}} \leqslant \epsilon_{1}
\end{aligned}
$$

since $\left\langle\epsilon_{L P} \mid \epsilon_{L P}\right\rangle=\epsilon_{L P_{1}}$ asymptotically. Thus for a chosen allowable error $\epsilon_{1}, \epsilon_{L P_{1}} \leqslant \epsilon_{1} / 2$. Hence from claim 2

$$
\gamma / \alpha \leqslant \sqrt{W\left(\frac{8}{\pi\left(0.01-\epsilon_{2}\right)^{2}}\right)}
$$

and so for a given stage 2 error $\epsilon_{2} \leqslant 0.01$ we can begin our two-stage dilution with a Lo-Popescu dilution with a classical communication cost no greater than

$$
2 \sqrt{W\left(\frac{8}{\pi\left(0.01-\epsilon_{2}\right)^{2}}\right)} \alpha_{\psi_{1}} \sqrt{\frac{N S\left(\psi_{2}\right)}{S\left(\psi_{1}\right)}} \text { bits. }
$$

A plot of the upper bound on $\gamma / \alpha$ noted in claim 2 against $\epsilon_{2}$ is given in Fig. 3 .

Applying the bound in [3] and using Eqs. (5) and (30), 


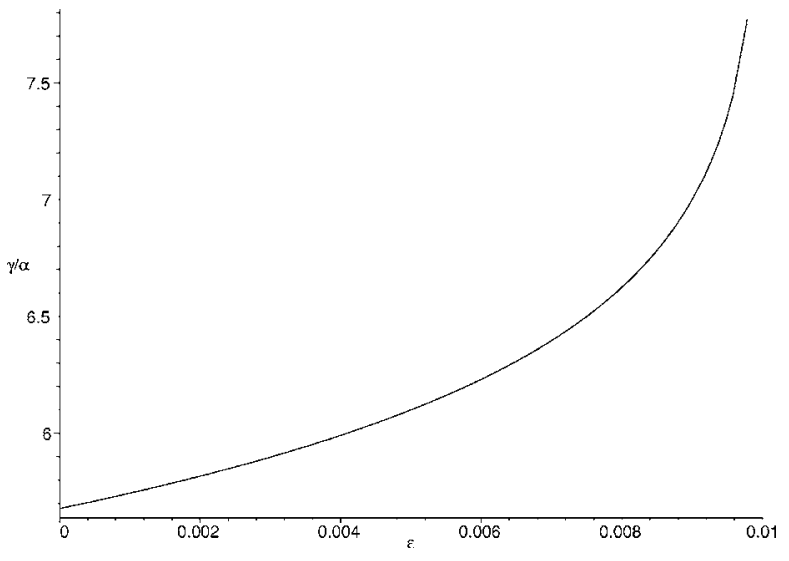

FIG. 3. Upper limit on $\gamma / \alpha$ as a function of $\epsilon_{2}$.

$$
\begin{gathered}
2 \sqrt{W\left(\frac{8}{\pi\left(0.01-\epsilon_{2}\right)^{2}}\right)} \alpha_{\psi_{1}} \sqrt{\frac{N S\left(\psi_{2}\right)}{S\left(\psi_{1}\right)}}+m \geqslant \alpha_{\psi_{2}} \sqrt{N}, \\
m \geqslant\left[\alpha_{\psi_{2}}-2 \sqrt{W\left(\frac{8}{\pi\left(0.01-\epsilon_{2}\right)^{2}}\right)} \alpha_{\psi_{1}} \sqrt{\frac{S\left(\psi_{2}\right)}{S\left(\psi_{1}\right)}}\right] \sqrt{N} \text { bits. }
\end{gathered}
$$

As one would expect, this bound is strictest for a dilution protocol with $\epsilon_{2}=0$, for which we have

$$
m \geqslant\left(\alpha_{\psi_{2}}-5.68 \alpha_{\psi_{1}} \sqrt{\frac{S\left(\psi_{2}\right)}{S\left(\psi_{1}\right)}}\right) \sqrt{N} \text { bits, }
$$

where the figure 5.68 is to two decimal points.

\section{THE INEFFICIENCY BOUND}

We can do an analogous calculation of the inefficiency in dilution between partially entangled states by considering the same two-stage dilution as before and the ebits lost at each stage, since we know that (ebits lost in Lo-Popescu dilution from singlets to $\left.\psi_{1}\right)+\left(\right.$ ebits lost in dilution from $\psi_{1}$ to $\left.\psi_{2}\right) \geqslant$ (the lower bound on inefficiency given in [3]) $=\alpha_{\psi_{2}} \sqrt{N}$ ebits.

\section{A. Inefficiency in the Lo-Popescu protocol}

In the Lo-Popescu protocol the only state that needs to be teleported is $\Delta$ and the inefficiency arises in this teleportation, which requires $\log _{2}[\operatorname{Sch}(\Delta)]$ ebits to teleport but which only provides $S(\Delta)$ ebits of shared entanglement.

We find that the entropy of those terms in $\epsilon_{L P_{2}}$ is asymptotically negligible (proportional to $2^{-(\omega-\gamma) \sqrt{N}}$ ), and hence that the entropy of $u_{1}$ is asymptotically just that of the typical set,

$$
\begin{aligned}
S\left(u_{1}\right) & =-\sum_{k_{\text {typical }}}\left(\begin{array}{l}
N \\
k
\end{array}\right) p^{k} q^{N-k} \log _{2}\left(p^{k} q^{N-k}\right) \approx \frac{-1}{\sqrt{2 \pi N p q}}\left[\left(N \log _{2} q\right) \int_{N p-\gamma \sqrt{N} / \log _{2}(q / p)}^{N p+\gamma \sqrt{N} / \log _{2}(q / p)} e^{-(k-N p)^{2} / 2 N p q} d k\right. \\
& =\frac{-1}{\sqrt{2 \pi}}\left[N \log _{2} q \int_{-\gamma / \alpha}^{\gamma / \alpha} e^{-x^{2} / 2} d x+N p \log _{2}(p / q) \int_{-\gamma / \alpha}^{\gamma / \alpha} e^{-x^{2} / 2} d x+\sqrt{N} p q \int_{-\gamma / \alpha}^{\gamma / \alpha} x e^{-x^{2} / 2} d x\right] \\
& =\frac{N S(\psi)}{\sqrt{2 \pi}} \int_{-\gamma / \alpha}^{\gamma / \alpha} e^{-x^{2} / 2} d x=N S(\psi)\left[1-\epsilon_{L P_{1}}\right],
\end{aligned}
$$

so the entanglement of normalized $u_{1}$ is asymptotically just $N S(\psi) . u_{1}=\Phi^{d} \otimes \Delta$, thus the entanglement of $\Delta$ is $N S(\Psi)$ $-d=\gamma \sqrt{N}$. So from Eq. (22) the asymptotic inefficiency (the number of ebits lost) in diluting from singlets to $N$ copies of $\psi$ via the Lo-Popescu protocol is

$$
\log _{2}[\operatorname{Sch}(\Delta)]-S(\Delta)=2 \gamma \sqrt{N}-\gamma \sqrt{N}=\gamma \sqrt{N} \text { ebits }
$$

Hence for the two-stage dilution, as described in Sec. IV, we can always choose our error in the Lo-Popescu protocol such that $\gamma / \alpha \leqslant \sqrt{W\left(8 / \pi^{2}\left(0.01-\epsilon_{2}\right)^{2}\right)}$ and so from the Harrow-Lo inefficiency bound we have that, for a loss of $k$ ebits from the unknown protocol,

$$
\sqrt{W\left(\frac{8}{\pi\left(0.01-\epsilon_{2}\right)^{2}}\right)} \alpha_{\psi_{1}}+k \geqslant \alpha_{\psi_{2}} \sqrt{N}
$$

$$
k \geqslant\left[\alpha_{\psi_{2}}-\sqrt{W\left(\frac{8}{\pi\left(0.01-\epsilon_{2}\right)^{2}}\right)} \alpha_{\psi_{1}}\right] \sqrt{N} \text { ebits }
$$

for an error $\epsilon_{2}$, with a coefficiency on $\alpha_{\psi_{2}} \sqrt{N}$ of 2.64 (to two decimal points), half of that in the classical communication cost.

\section{THE GENERAL PURE STATE CASE}

For the general pure state

$$
|\psi\rangle=\sum_{i=1}^{b} \sqrt{p_{i}}|i i\rangle
$$

we can perform an analogous analysis to the single-qubit case, bounding the degeneracy of terms in $\psi^{N}$ 


$$
N S(\psi)-\gamma \sqrt{N} \leqslant \log _{2}\left(\begin{array}{c}
N \\
k_{1} \ldots k_{b}
\end{array}\right) \leqslant N S(\psi)+\gamma \sqrt{N}
$$

We use the substitution (from [10], though it mistakenly quotes $\pi_{i}^{-1}$ instead of $\pi_{i-1}^{-1}$ in the numerator) of

$$
y_{i}=\frac{k_{i}-\left(N-k_{i}-\ldots-k_{i-1}\right) p_{i} \pi_{i-1}^{-1}}{\sqrt{N p_{i} \pi_{i} \pi_{i-1}^{-1}}}, \quad \pi_{i}=1-p_{1}-\ldots p_{i}
$$

giving the asymptotic approximation

$$
\left(\begin{array}{c}
N \\
k_{1} \ldots k_{b}
\end{array}\right) p_{1}^{k_{1}} \ldots p_{b}^{k_{b}} \sim \frac{e^{-\left(y_{1}^{2}+\ldots+y_{b-1}^{2}\right) / 2}}{(\sqrt{2 \pi N})^{b-1} \sqrt{p_{1} p_{2} \ldots p_{b}}}
$$

Differentiating the degeneracies with respect to $y_{i}$, we find that for the typical set

$$
\left|\sum_{i=1}^{b-1} \Omega_{i} \Delta y_{i}\right| \leqslant \gamma
$$

where

$$
\Omega_{i}=\sqrt{\frac{p_{i}}{\pi_{i} \pi_{i-1}}} \sum_{j=i+1}^{b} p_{j} \log _{2}\left(p_{j} / p_{i}\right)
$$

We are unaware of any expression for the integral of the probability distribution (41) over the range (42). Instead we choose an expression $\Omega_{t} \geqslant\left|\Omega_{i}\right| \forall i<b$ and integrate (4) outside the range $\pm \gamma / \Omega_{\max }$ for all $y_{i}$ to obtain an upper bound on $\epsilon_{L p_{1}}$. Doing so we find

$$
\begin{aligned}
\epsilon_{L p_{1}} & \leqslant 2\left(\frac{1}{\sqrt{2 \pi}} \int_{-\gamma / \Omega_{t}}^{\gamma / \Omega_{t}} e^{-y^{2} / 2} \mathrm{dy}\right)^{b-1} \\
& \leqslant \frac{2}{\left[\sqrt{2 \pi}\left(\gamma / \Omega_{t}\right)\right]^{b-1}} e^{-(b-1)\left(\gamma / \Omega_{t}\right)^{2}}
\end{aligned}
$$

which inverts to

$$
\frac{\gamma}{\Omega_{t}} \leqslant \sqrt{W\left(\frac{1}{2 \pi\left(\epsilon_{L p_{1}} / 2\right)^{2 /(b-1)}}\right)}
$$

\section{Inefficiency and classical communication bounds for the general pure state}

The remainder of the analysis follows as for the singlequbit case-in particular we find that Claims 1 and 2 still apply (with (46) substituted into Claim 2) and our final results are, that for an $m$-bit classical communication cost and $k$-ebit inefficiency diluting from $\psi_{1}$ to $\psi_{2}$, where $\psi_{1}$ has Schmidt number $b$

$$
m \geqslant \alpha_{\psi_{2}}-2 \sqrt{W\left(\frac{1}{2 \pi\left(\frac{0.01-\epsilon_{2}}{4}\right)^{2 /(b-1)}}\right)} \Omega_{t \psi_{1}} \sqrt{\frac{S\left(\psi_{2}\right)}{S\left(\psi_{1}\right)}} \sqrt{N}
$$

$$
\begin{aligned}
k \geqslant & \alpha_{\psi_{2}}-\sqrt{W\left(\frac{1}{2 \pi\left(\frac{0.01-\epsilon_{2}}{4}\right)^{2 /(b-1)}}\right)} \Omega_{t \psi_{1}} \\
& \left.\times \sqrt{\frac{S\left(\psi_{2}\right)}{S\left(\psi_{1}\right)}}\right) \sqrt{N}
\end{aligned}
$$

\section{CONCLUSION}

We have derived lower bounds for the classical communication cost and inefficiency of converting between pure bipartite entangled states expressible in two terms. Specifically, we have proven the following results.

Claim 3. For any protocol which converts via local operations and classical communication $N S\left(\psi_{2}\right) / S\left(\psi_{1}\right)$ copies of a state $\psi_{1}$ into $N$ copies of state $\psi_{2}$ with at most error $\epsilon_{2}$ $<0.01$, in the limit of large $N$ and where $\psi_{1}, \psi_{2}$ are bipartite pure entangled states and $\psi_{1}$ is expressible in two terms.

$$
\left[\alpha_{\psi_{2}}-2 \sqrt{W\left(\frac{8}{\pi\left(0.01-\epsilon_{2}\right)^{2}}\right)} \alpha_{\psi_{1}} \sqrt{\frac{S\left(\psi_{2}\right)}{S\left(\psi_{1}\right)}}\right] \sqrt{N} \text { bits. }
$$

Claim 4. For the protocol described in claim 3, the inefficiency is at least

$$
\left[\alpha_{\psi_{2}}-\sqrt{W\left(\frac{8}{\pi\left(0.01-\epsilon_{2}\right)^{2}}\right)} \alpha_{\psi_{1}} \sqrt{\frac{S\left(\psi_{2}\right)}{S\left(\psi_{1}\right)}}\right] \sqrt{N} \text { ebits. }
$$

Proof. As shown in Secs. III-V.

These bounds are meaningful in that they give nonzero classical communication costs for conversions between possible states; however, they only give a partial ordering on entangled states due to the asymmetry of the coefficients involved. Namely, nonzero classical communication is required for entanglement conversion from $\psi_{1}$ to $\psi_{2}$ when

$$
\frac{\alpha_{\psi_{2}}}{S\left(\psi_{2}\right)} \geqslant 2 \sqrt{W\left(\frac{8}{\pi\left(0.01-\epsilon_{2}\right)^{2}}\right)} \frac{\alpha_{\psi_{1}}}{S\left(\psi_{1}\right)}
$$

and nonzero inefficiency when

$$
\frac{\alpha_{\psi_{2}}}{S\left(\psi_{2}\right)} \geqslant \sqrt{W\left(\frac{8}{\pi\left(0.01-\epsilon_{2}\right)^{2}}\right)} \frac{\alpha_{\psi_{1}}}{S\left(\psi_{1}\right)} .
$$

So, for example, for a protocol with essentially zero error, there will be a nonzero classical communication cost in diluting, e.g., states $\left|\psi_{1}\right\rangle=\sqrt{0.43}|00\rangle+\sqrt{0.57}|11\rangle$ to states $\left|\psi_{2}\right\rangle$ $=\sqrt{0.14}|00\rangle+\sqrt{0.86}|11\rangle$, and a nonzero inefficiency in diluting the same $\psi_{1}$ to states $\left|\psi_{2}\right\rangle=\sqrt{0.3}|00\rangle+\sqrt{0.7}|11\rangle$. That is, these bounds do give usable limits on allowable dilutions.

We have also derived explicit expressions for various details of the Lo-Popescu protocol, in particular the dependence of the coefficient in the $O(\sqrt{N})$ classical communication cost on the allowable error. 
There is, however, no reason to believe that these bounds cannot be improved-their derivation is dependent on considering one particular entanglement dilution protocol (LoPopescu) and a tighter bound could potentially be derived by considering a different protocol with lower inefficiency and classical communication costs.

\section{General pure states}

For dilution of general pure states, we have found:

Claim 5. For any protocol which converts via LOCC $N S\left(\psi_{2}\right) / S\left(\psi_{1}\right)$ copies of a state $\psi_{1}$ into $N$ copies of state $\psi_{2}$ with at most error $\epsilon_{2}<0.01$, in the limit of large $N$ and where $\psi_{1}, \psi_{1}$ are bipartite pure entangled states and $\psi_{1}$ is of Schmidt number $b$, the classical communication cost is at least

$\left(\alpha_{\psi_{2}-2} \sqrt{W\left(\frac{1}{2 \pi\left(\frac{0.01-\epsilon_{2}}{4}\right)^{2 / b-1}}\right)} \Omega_{t \psi_{1}} \sqrt{\frac{S\left(\psi_{2}\right)}{S\left(\psi_{1}\right)}}\right) \sqrt{N}$ bits.

for $\Omega_{t \psi_{1}} \geqslant\left|\Omega_{i \psi_{1}}\right| \forall i<b$.

Claim 6. For the protocol described in Claim 5, the inefficiency is at least

$\left(\alpha_{\psi_{2}}-\sqrt{W\left(\frac{1}{2 \pi\left(\frac{0.01-\epsilon_{2}}{4}\right)^{2 / b-1}}\right)} \Omega_{t \psi_{1}} \sqrt{\frac{S\left(\psi_{2}\right)}{S\left(\psi_{1}\right)}}\right) \sqrt{N}$ ebits.
Choosing $\Omega_{t}$

Still undefined is how one chooses $\Omega_{t}$. Ideally one would take $\Omega_{t}$ equal to the largest $\Omega_{i}$, but it can be seen from (6) that the values of $\Omega_{i}$ are dependent on the arbitrary ordering of the coefficients $p_{i}$. Thus the tightest bound this analysis provides is for $\Omega_{t}$ equal to the maximum $\Omega_{i}$ for the ordering in which this maximum is smallest. For a given state this is certainly a well-defined quantity, but we do not have a general formula for it in terms of the $p_{i}$ 's.

An alternative prescription for $\Omega_{t}$ is to order $p_{1} \leqslant p_{2}$ $\leqslant \ldots \leqslant p_{b}$ and use

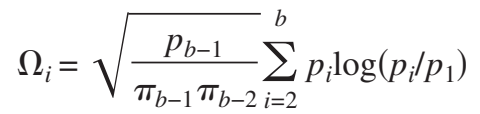

which is simply expressed, but depending on the state may provide a much looser bound. Whatever the chosen $\Omega_{t}$, using a single bound for all the $y_{i}$ clearly makes the overall bound looser than in the two Schmidt term case.

However, even the general $\Omega_{t}$ in (55) can give specific bounds on classical communication and inefficiency. E.g., we find that for an error-free protocol diluting from many copies of $\sqrt{0.3}|11\rangle+\sqrt{0.3}|22\rangle+\sqrt{0.4}|33\rangle$ to $\sqrt{0.1}|11\rangle$ $+\sqrt{0.1}|22\rangle+\sqrt{0.8}|33\rangle$ at least $0.29 \sqrt{N}$ bits of classical communication are required and at least $0.87 \sqrt{N}$ ebits are lost. It may well be feasible to obtain much tighter bounds in specific cases.

\section{ACKNOWLEDGMENTS}

Support of the funding agencies CFI, CIPI, the CRC program, NSERC, OIT, and PREA is gratefully acknowledged.
[1] C. H. Bennett, H. J. Bernstein, S. Popescu, and B. Schumacher, Phys. Rev. A 53, 2046 (1996).

[2] H. K. Lo and S. Popescu, Phys. Rev. Lett. 83, 1459 (1999).

[3] A. Harrow and H. K. Lo, IEEE Trans. Inf. Theory 50, 319 (2004).

[4] P. Hayden and A. Winter, Phys. Rev. A 67, 012326 (2003).

[5] W. Feller, An Introduction to Probability Theory and Its Applications (John Wiley \& Sons, New York, 1957) p. 170.

[6] R. Corless, G. Gonnet, D. Hore, D. Jeffrey, and D. Knuth, Adv. Comput. Math. 5, 329 (1996).
[7] T. Worsch, Universität Karlsruhe, Fakultät für Informatik, Technical Report No. 31/94, 1994 (unpublished).

[8] H. K. Lo, Phys. Rev. A 62, 012313 (2000).

[9] M. Nielsen and I. Chuang, Quantum Computation and Quantum Information (Cambridge University Press, Cambridge, U.K., 2000), p. 406.

[10] N. K. Arenbaev, Asymptotic behavior of the multinomial distribution, Theory of Probability and is Applications, 4 Vol. XXI (1976). 